\title{
Liquid-Solid Phase Transition of the System with Two particles in a Rectangular Box.
}

\author{
Akinori Awazu* \\ Department of Mathematical Sciences \\ Osaka Prefecture University, Sakai 599-8531 Japan.
}

\begin{abstract}
We study the statistical properties of two hard spheres in a two dimensional rectangular box. In this system, the relation like Van der Waals equation loop is obtained between the width of the box and the pressure working on side walls. The autocorrelation function of each particle's position is calculated numerically. By this calculation near the critical width, the time at which the correlation become zero gets longer according to the increase of the height of the box. Moreover, fast and slow relaxation processes like $\alpha$ and $\beta$ relaxations observed in supper cooled liquid are observed when the height of the box is sufficiently large. These relaxation processes are discussed with the probability distribution of relative position of two particles. PACS number(s):
\end{abstract}

Liquid-solid phase transition is one of the most familiar phenomena for us. This transition of a system with so many degrees of freedom has been studied through many kinds of analytical models and numerical model\$1. In numerical parts, the liquid-solid phase transition is observed in the system containing $10^{1 \sim 4}$ hard or soft core particles by MonteCalro simulation and Molecular Dynamics simulation日 2 $^{6}$. The motions of individual particles

\footnotetext{
*E-mail: awa@zenon.ms.osakafu-u.ac.jp
} 
(moleculars) are different between in liquid phase and in solid phase as follows. In the liquid phase, particles can exchange their own positions each other, and each particle can wander all around the system. On the other hand, in the solid phase, particles cannot exchange their own positions, and they move only restricted small areas.

Now we consider a rectangular box containing two hard spheres with same diameter $d$. The height of the box is larger than $2 d$. When the width of the box is larger than $2 d$, these spheres can exchange their own positions (Fig.1 (a)). However, these particles cannot exchange their positions when the width of box is smaller than $2 d$ (Fig.1 (b)). From these facts, we regard the former state and the latter state as the simplest forms of, respectively, the liquid state and the solid state. Then, a problem arises; In such a simple system near the critical width $(=2 d)$ of the box, can we find characteristic phenomena like Alder transition 3 日. 6 of the system with many hard spheres? In this paper, we focus on statistical and dynamical properties of the spheres near the critical width of the box to understand this problem.

The system on our consideration consists of two dimensional hard sphere particles with unit mass and unit radius which are confined in a two-dimensional rectangular box. Here, the width and the height of the box are respectively $a$ and $b$, and all the walls are rigid (Fig.1). The interaction between two particles or between a particle and a wall, is only through hard core collisions. These tasks are implemented in the following manner; the tangential velocities to the collision plane are preserved, while the normal component of relative velocity $\Delta v_{n}$ changes into $-\Delta v_{n}$. The total energy of this system is given as 1 . Because this system consists of rigid spheres and rigid walls, the qualitative behaviors are independent of the total energy. We set $b>4$ for most of our discussions which means these two particles can exchange their positions in horizontal direction with each other. Note, in this paper, the state with $a>4$ is regarded as the liquid state and the state with $a<4$ is regarded as the solid state.

Figure 2 (a) and (b) are typical relations between the width $a$ and the pressure $P_{a}$ which works on side walls for each height $b=4.3 \sim 6.7$. Here, $P_{a}$ is defined as the time average of 
the impulses caused by the bouncing of particles on side walls per unit length per unit time. In these figures, the region of $a$, in which the volume compressibility is negative, appears for small $b$ around the critical width $a^{*}=4.0$. These curvatures are similar to the Van

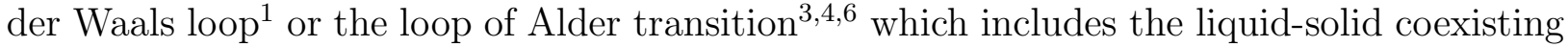
region. This negative volume compressibility seems to indicate the appearance of phase transition around the critical width $a^{*}=4.0$ which distinguishes the solid state from the liquid state. If $b$ becomes larger than a critical value $b^{*} \sim 6.0$, however, the form of this curvature is loosened, and the compressibility becomes positive for all $a$. In this case, we cannot observe the distinction between liquid state and solid state. Figure 2 (c) and (d) are the typical relations between the width $a$ and the pressure which works on upper and lower walls $P_{b}$ for each height $b=4.3 \sim 6.7$. Here, $P_{b}$ is defined as the time average of the impulses caused by the bouncing of particles on upper and lower walls per unit length per unit time. Different from the relation between $a$ and $P_{a}, P_{b}$ decrease monotonically with the increase of $a$. Such anisotropy seems one of the characteristic features of this system, which doesn't appear in the system with many hard spheres. If we focus only on the $a-P_{a}$ relation, however, this system can be regarded as one of the simplest model to imitate phenomena of the liquid-solid phase transition. Here, $a$ and $P_{a}$ correspond to, respectively, the volume and pressure of the system with many particles.

Now, we consider the counterpart of the above $b$ in the system with many hard spheres or in more general systems with many degrees of freedom. Figure 3 (a) is a typical autocorrelation functions of the position of each particle $C(t)=<\mathbf{x}(0) \mathbf{x}(t)>/<\mathbf{x}(0) \mathbf{x}(0)>$ in the solid state $(a=3.8)$ and liquid states $(a=4.1, a=4.5$, and $a=5.0$ with $b=5.5)$. Here, $\mathbf{x}(t)$ is the position of a particle. In the liquid state, the relaxation process becomes slower as the width comes closer to the critical value. In the solid state $(a=3.8)$, the correlation function has a finite value for $t \rightarrow \infty$ because two particles cannot exchange their positions. Figure 3 (b) is the auto-correlation functions for $b=4.3,5.1,5.9,6.7$ near the critical width $a^{*}(a=4.1)$. These curvatures indicate that each relaxation process contains the fast and the slow relaxation processes for a little over the critical width. These 
fast and slow relaxations can be fitted with functions, respectively, $t^{-\beta}(\beta \sim 0.75)$ and $\exp \left(-t^{\alpha}\right)(\alpha \sim 0.67)$. By increasing $b$, the form of $C(t)$ changes as follows. i) The time at which the correlation becomes zero gets longer. ii) When $b$ is larger than a critical value $b^{* *} \sim 5.1$, the fast relaxation and the slow relaxation are separated clearly by the appearance of plateau. These relaxations are respectively similar to $\beta$ and $\alpha$ relaxations of the density fluctuations in super cooled liquidt. The system which includes non-uniform moleculars tends to become super cooled liquid state when it is cooled or compressed 7 . Moreover, the liquid-solid coexisting region disappears in the system with many hard spheres when the size polydispersity of spheres is larger than a critical value日. From these facts, we expect that present two particles system imitates the phase transition of a system which consists of many non-uniform elements. Here, the quantity $b$ corresponds to the dispersion of particles' characters like the size polydispersity in the system with many particles. In order to discuss the mechanism of above simulation results, we focus on the statistical properties of each particle's trajectory for each $b$ near the width $a^{*}$.

Figure 4 (a), (b) and (c) are typical trajectories of centers of particles for, respectively, $b=4.7, b=5.7$ and $b=6.7$ with $a=4.1$. If the volume of the box is enough large and we can ignore the particles' volume, these trajectories fill the rectangular region which is enclose by points $\left(\frac{a-d}{2}, \frac{b-d}{2}\right),\left(\frac{a-d}{2},-\frac{b-d}{2}\right),\left(-\frac{a-d}{2}, \frac{b-d}{2}\right)$ and $\left(-\frac{a-d}{2},-\frac{b-d}{2}\right)$ (Fig.4(c)). Here, $d$ is the diameter of each particle which is set as 2 in our discussion. This means particles wander all around the box like ideal gas systems. When the size of box becomes small, however, the finite volume effect of particles become evident. Particularly, if the relation

$(a-d)^{2}+(b-d)^{2}<(2 d)^{2}$

is satisfied, inhibited region for particles' center to come in appears around the central part of the box. When $b<2+2 \sqrt{2}$ is satisfied, the above equation is satisfied around $a=a^{*}=4$ and the trajectory of the center of particle is given like Fig.4 (a). This is also the case for $a<a^{*}=4$ where the trajectory of the center of particle is given like Fig.4 (b) if $2+2 \sqrt{2}<b<b^{*}=6$. In these cases, each trajectory of the center of particle is similar to 
what is observed in Sinai billiard 8 . Thus, in order to discuss these situations, we consider the $(a-d) \times(b-d)$ rectangular Sinai billiard which includes a hard sphere with diameter $d(d=2)$. Using the equi-partition rule, we can calculate the $a-P_{a}$ relation of Sinai billiard analytically like correlated cell modele . For each particle in Sinai billiard, the entropy $S$ is given by using the phase space volume $S=\log \left(A_{r}(a, b) A_{v}\right)$ and free energy $F$ is given as $F=U-S$. (Boltzmann constant $k_{B}$ and temperature $T$ are set as 1.) Here, $A_{r}(a, b)$ and $A_{v}$ are phase space volumes of, respectively, real space part and velocity space part, and internal energy $U$ is constant because this system consists of hard walls and a hard sphere. $A_{r}(a, b)$ is given as

$A_{r}=(a-d)(b-d)-\pi \cdots(a>4)$

$A_{r}=(a-d)(b-d)-(a-d) \cos \theta-2 \theta \cdots(a \leq 4)$

where $\sin \theta=\frac{a-d}{d}$ and $d=2$. Using the above relations also with $P_{a} b=-\frac{\partial F}{\partial a}, a-P_{a}$ relation of the system for $b<6$ is obtained, and we can observe the liquid-solid phase transition like Fig. 5(a). In this case, the width $a=a^{*}=4$ is a singular point and this point gives the maximal pressure independent of $b$. On the other hand, however, the $a-P_{a}$ relation in simulation results (Fig.2 (a) and (b)) have the inflection point near the critical width $a=a_{*}=4$, and the form of each curvatures is smooth. In addition, we consider a square box system in which $a$ and $b$ are varied with $a=b$. This system satisfies $b<2+2 \sqrt{2}$ around $a \sim a^{*}$. In the same way of the calculation of above rectangular Sinai billiard, the $a-P_{a}$ relation of the $(a-d) \times(a-d)$ square Sinai billiard can be obtained analytically. In this calculation, the profile of the $a-P_{a}$ relation of the square Sinai billiard is obtained as the almost same one as the results of the rectangular billiard. However, it is remarkable that $P_{a}\left(=P_{b}\right)$ decreases monotonically with increasing $a(=b)$ in the simulation of two particles in the square box (Fig.5 (b)).

Finally, we discuss the mechanism of the appearance of plateau in $C(t)$, the autocorrelation function of each particle's position, near the width $a=a^{*}$ considering statistical 
properties of particles' trajectories. Now, we define $\operatorname{Pro}_{\delta x}$ and $\operatorname{Pro}_{\delta y}$ as the probability distributions of $\delta x$ and $\delta y$. Here, $\delta x$ is horizontal component and $\delta y$ is vertical component of relative position vector between centers of two particles. Figure 6 (a) and (b) are respectively $\delta y$-Pro $\operatorname{Pry}_{y}$ relations and $\delta x$-Prosx relations of the case $b=4.3,4.7,5.1,5.5,5.9$ with $a=4.1$. In Fig.6 (a), the maximum points of $\operatorname{Pro}_{\delta y}$ are always far from the point $\delta y=0$. In Fig.6 (b), however, the position of the maximum point of $\operatorname{Pro}_{\delta x}$ depends on $b$. When $b$ is small, the maximum points of $\operatorname{Pro}_{\delta x}$ are far from the point $\delta x=0$. This means that two particles tend to face each other on a diagonal line of box. By this tendency, it is rather easy for these two particles to exchange their positions both in vertical and horizontal direction. Hence, the time at which $C(t)$ become 0 is relatively short. On the other hand, only one maximum point of $\operatorname{Pro}_{\delta x}$ appear at $\delta x=0$ when $b$ is larger than the critical value $b^{* *} \sim 5.1$. In this case, the height of box is enough large to change particle's positions almost freely but only in the horizontal direction. This is considered as the origin of fast relaxation in the simulation. This situation also means that two particles, in statistical sense, tend to line up on a vertical line. In this case, the exchange of two particles' positions in vertical direction is cut across strongly. This fact originates the appearance of the plateau in $C(t)$, after which the slow relaxation starts.

In this paper, liquid-solid phase transition and the long time correlation of two hard spheres confined in a two dimensional rectangular box is studied. Between the width of the box and the pressure at the side walls, the relation like Van der Waals equation is obtained. However, the range of the box width, in which the volume compressibility is negative, disappears when the height of this box passes through a critical value. The auto-correlation function of each particle's position is calculated near the critical width. According to the increase of the height of this box, the time at which this correlation become zero gets longer. Moreover, a fast relaxation and a slow relaxation are separated clearly by the appearance of plateau when the height of this box is sufficiently large. These relaxation processes are discussed considering the form of the probability distribution of relative position of two particles. As a conclusion, this system is considered to be one of the simplest system which 
imitates the liquid-solid phase transition of the system which has been believed to be a characteristic nature of systems with many non-uniformed elements. Still, for the relation between the width of the box and the pressure at the side walls, some discrepancies appear between the analytical and simulation results. Thus further consideration is required on the dynamical properties like the long time correlation which forbids the equi-partition. These topics seem to have strong relation with the slow dynamics in Hamilton dynamical systems10. In addition, the pressure which works on walls is anisotropic in our system, while the pressure of the system with many particles is usually uniform. This also is a problems to be solved. Moreover, the understanding of the glass transition or the other non-equilibrium system 11 through our simple model is one of future issues.

The author is grateful to H.Nishimori, N.Ito, S.Sasa, H.Hayakawa, M.Sano and K.Sekimoto for useful discussions. This research was supported in part by the Ibaraki University SVBL and Grant-in-Aid for JSPS Felows 10376. 


\section{REFERENCES}

1. H.E Stanley, INTRODUCTION TO PHASE TRANSITIONS AND CRITICAL PHENOMENA. Clarendon Press Oxford (1971); N.E Cusack, THE PHYSICS OF STRUCTURALLY DISORDERED MATTER, University of Sussex Press;J.M. Ziman, MOREL OF DISORDER. Syndics of the Cambridge Press (1979); W.G Hoover, Computational Statistical Mechanics, Elsevier Science B. V. (1991)

2. W.W. Wood and J.D. Jacobson, J. Chem. Phys 27 (1957) 1207; B.J Alder and T.E Waiwright, J. Chem. Phys 27 (1957) 1208; J. Chem. Phys 33 (1960) 1439; W.G Hoover and R.M Ree J. Chem. Phys 49 (1968) 3609

3. B.J Alder and T.E Waiwright, Phys. Rev. 127 (1962) 359

4. J.A. Zollweg and G.V. Chester, Phys. Rev. B46 (1992) 11186; J. Lee and K.J. Strandburg, Phys. Rev. B46 (1992) 11191

5. J.P Hansen and L Verlet Phys. Rev. 184 (1969) 151;

6. W. Vermhlen and N. Ito Phys. Rev. E51 (1995) 4325

7. For example, Science 267 (1995) 1924,1935,1939,1945 and 1947 M.D. Edinger, C.A. Angell and S.R. Nagel, J. Phys. Chem. 100 (1996) 13200 J Matsui, T Odagaki and H Hiwatari, Phys. Rev. Lett 73 (1994) 2452; R Yamamoto and A Onuki, Phys, Rev. E. 58 (1998) 3515; W Kob et al., Phys. Rev. Lett 79 (1997) 2827

8. G.M. Zaslavsky, Chaos in Dynamical System (Harwood Academic, New York, 1985)

9. B.J. Alder, W.G. Hoover and T.E. Wainwright, Phys.Rev.Lett 11 (1963) 241; Dh. Dellago and H.A. Posch, Physica A 230 (1996) 364

10. Y Aizawa, Prog. Theor. Phys.,71 (1984) 1419; G Benettin, Prog. Theor. Phys. Suppl, 116 (1994) 207; G Benettin, L Galgani and A Giorgilli Phys. Lett A 120 (1987) 23; Y.Y Yamaguchi and T Konishi, Prog. Theor. Phys.,99 (1998) 139; Y.Y Yamaguchi, Moder- 
ately Chaotic Hamiltonian System: Structure and Motion in High-dimensional Phase Space, Ph.D thesis of Nagoya University; L Meza-Montes and S E. Ulloa, Phys,Rev. E. $55(1997) 6319$

11. Dh. Dellago and H.A. Posch and W.G. Hoover, Phys,Rev. E. 53 (1996) 1485 


\section{FIGURES}

Fig. 1. Illustration of two particles system in rectangular box. (a)Width of box is larger than sum of two diameters (Liquid state), and (b)width of box is smaller than sum of two diameters (solid state).

Fig. 2. Relations between the width $a$ and the pressure $P_{a}$ with (a) $b=4.3,4.5,4.7,4.9$ (b) $b=5.1,5.5,5.9,6.3,6.7$ in descending order, and that between the width $a$ and the pressure $P_{b}$ with (c) $b=4.3,4.5,4.7,4.9$ (d) $b=5.1,5.5,5.9,6.3,6.7$.

Fig.

3.

Auto-correlation

function of each particle's position $C(t)$, respectively, (a) $a=3.8,4.1,4.5,5.0$ with $b=5.5$, and (b) $b=4.3,5.1,5.9,6.7$ with $a=4.1$. Fitting lines are $\propto t^{-0.75}$ and $\propto \exp \left(-t^{0.67}\right)$.

Fig. 4. Typical trajectories of particles respectively (a) $b=4.7,(\mathrm{~b}) b=5.7$ and $(\mathrm{c}) b=6.7$ with $a=4.1$.

Fig. 5. (a) $a-P_{a}$ relations of rectangular Sinai billiard with $b=4.5,5.1$ and 5.7, and (b) $a-P_{a}$ relations of two particles in square box.

Fig. 6. Probability distributions (a)Pro $\delta y$ of vertical component of relative position vector $\delta y$, and (b) $\operatorname{Pro}_{\delta x}$ of horizontal component of relative position vector $\delta x$ between two particles with $a=4.1 . b=4.3,4.7,5.1,5.5$ and 5.9 from the bottom up near $\delta x=0.0$ in (b). 

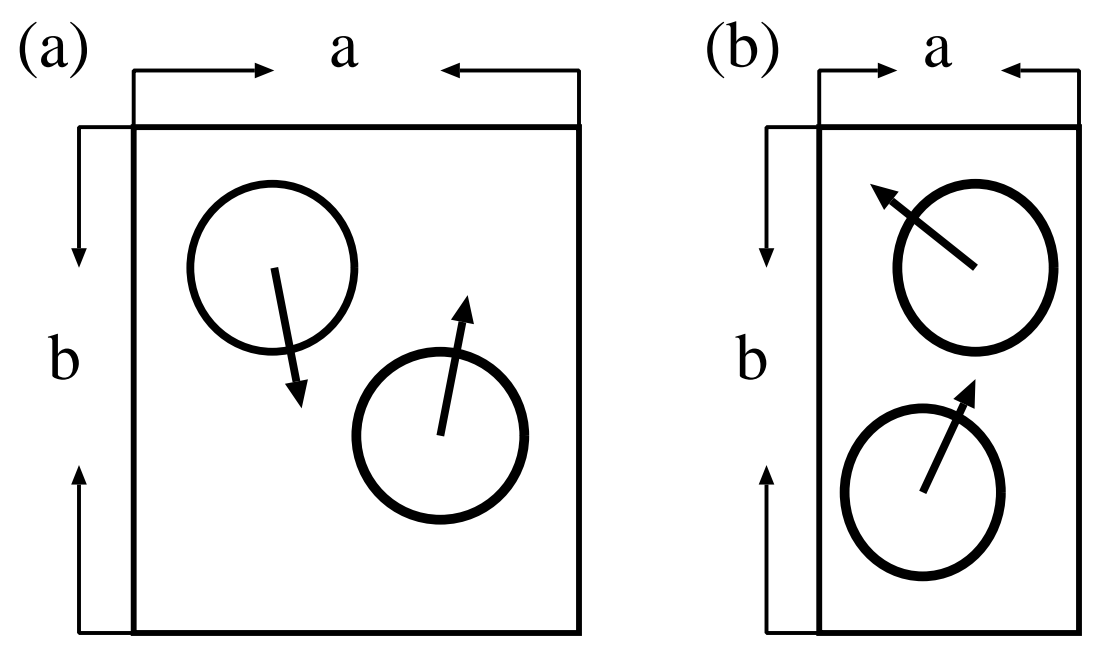

A Awazu Figure 1 

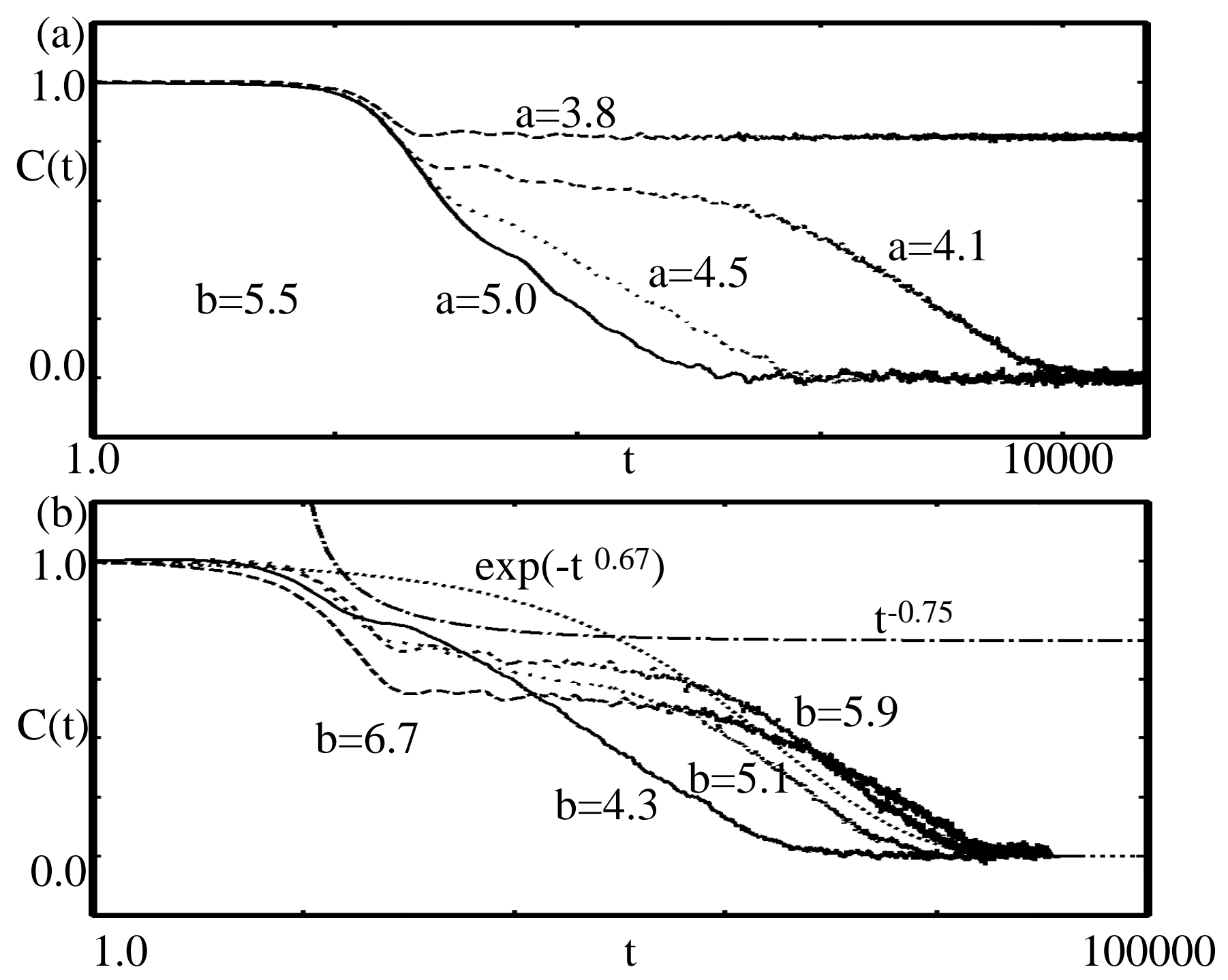

A Awazu Figure 3 


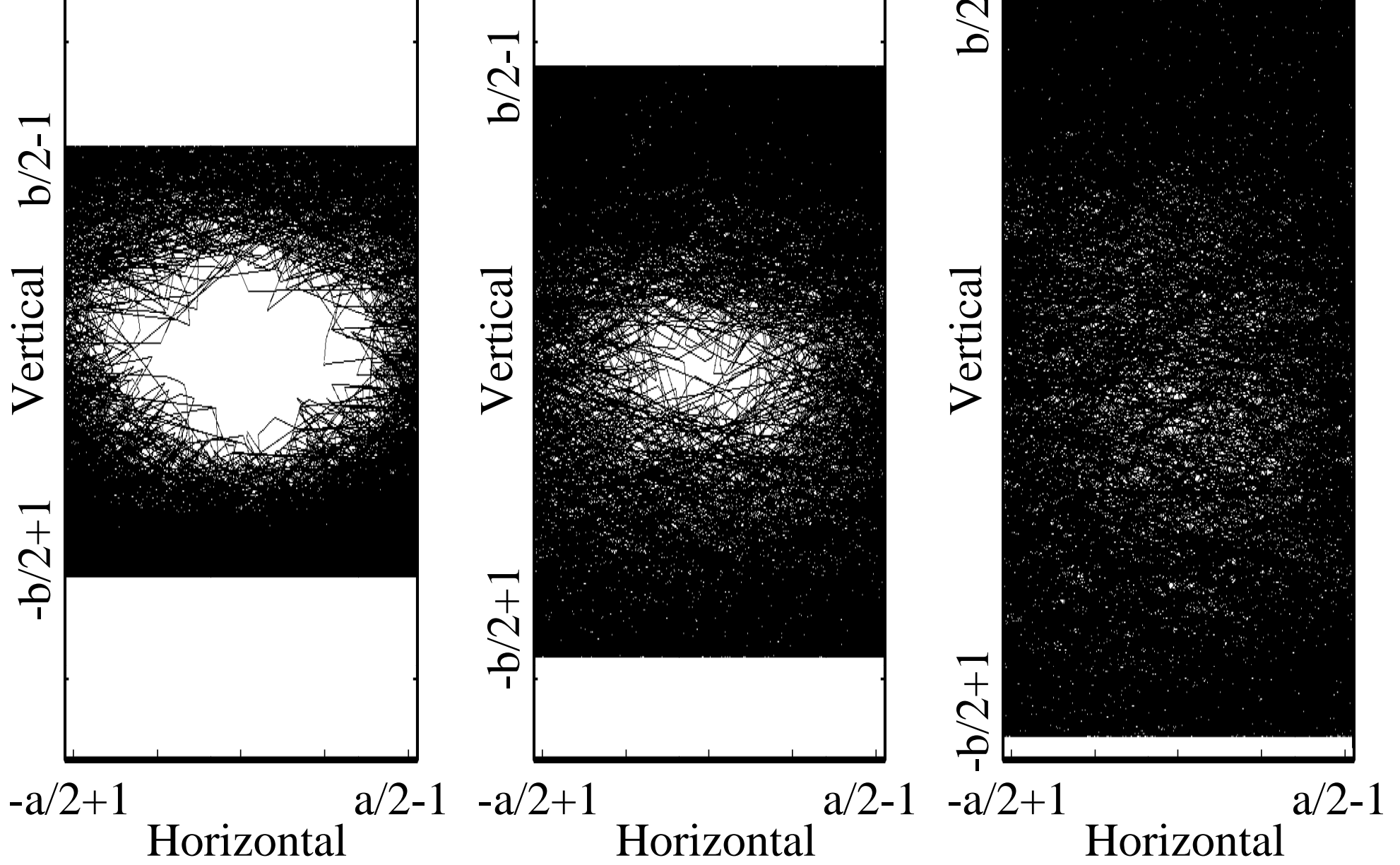

A Awazu Figure 4 

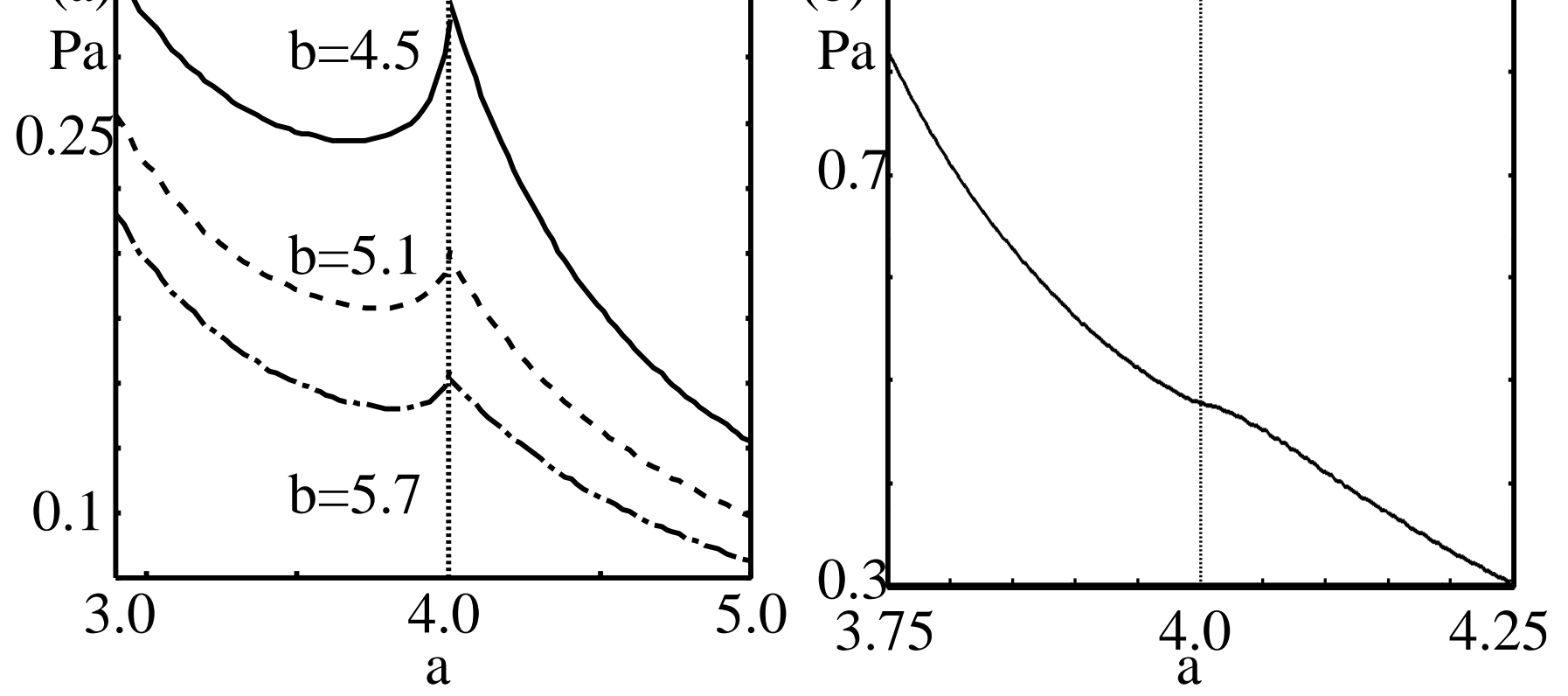

A Awazu Figure 5 


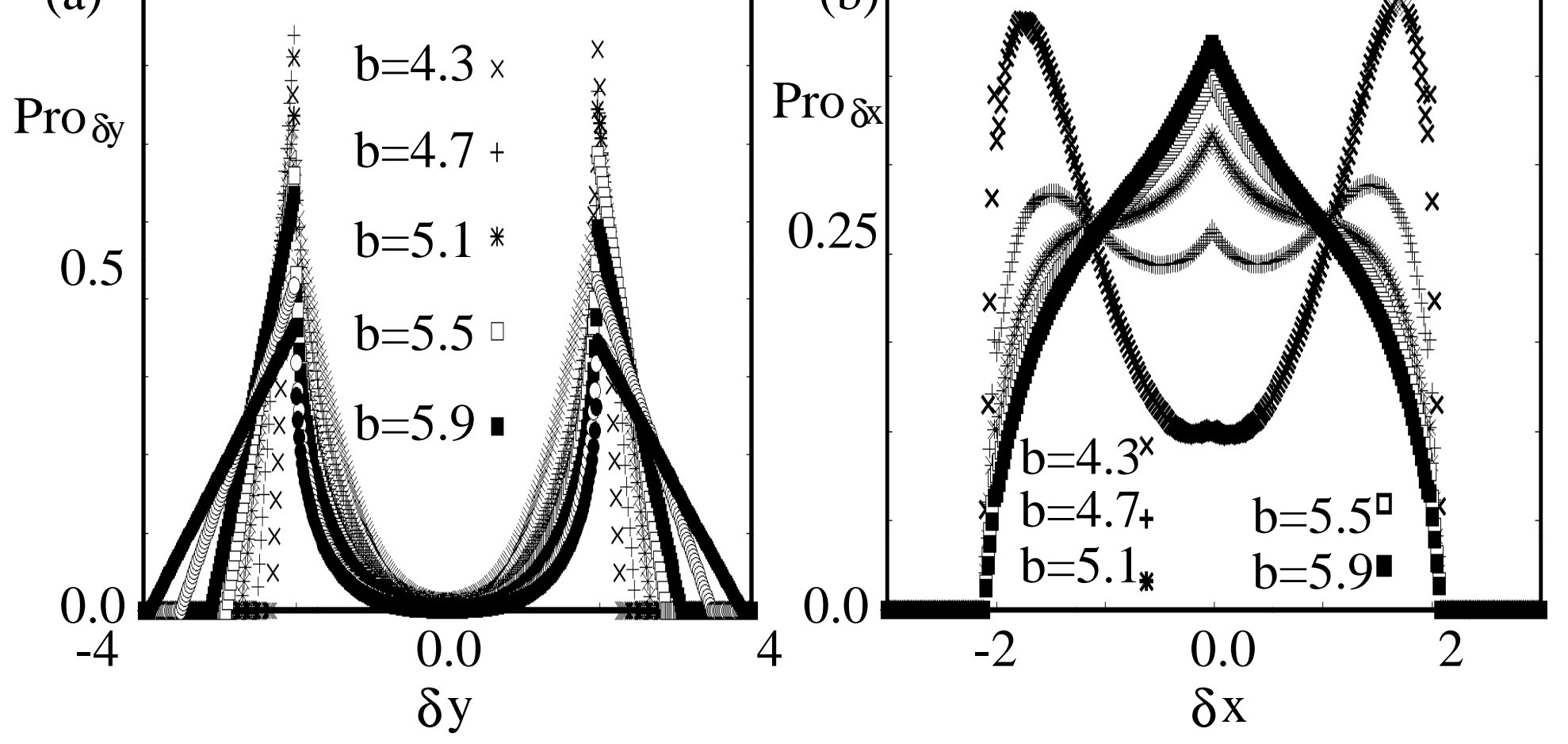

A Awazu Figure 6 